linked to 3 possibilities: 1) febrile seizures are the first manifestation of epilepsy; 2) they are an age-specific marker of inherent susceptibility to seizures, and 3) prolonged febrile seizures (complex FS) may damage the brain with consequent increased risk of seizures. (Vestergaard M et al. Am J Epidemiol 2007;165:911-918). In the Collaborative Perinatal Project, $1 \%$ of children with simple febrile seizures had developed epilepsy by age 7 years. In children with complex febrile seizures, the risk was $9.2 \%$, and in the total cohort, the risk was $2 \%$. In children with no febrile seizures, the risk is $0.5 \%$. The risk of epilepsy at 7 years of age in children with simple febrile seizures is two times higher, and in those with complex febrile seizures it is 18 times higher than in children with no febrile seizures. (Nelson KB, Ellenberg JH. Pediatrics 1978;61:720-727).

Febrile seizure inheritance and SUD in toddlers. SUD in 6 toddlers reported from Children's Hospital, Boston was associated with an autosomal dominant inheritance of febrile seizures, and with hippocampal abnormalities in one of 3 autopsied cases. The autosomal dominant pattern of inheritance for febrile seizures in affected families was identical to that observed in genetic epilepsy with febrile seizures plus and familial febrile seizures. Febrile seizure may be a marker of a process that leads to SUD, and seizure may or may not be directly involved. Genetic markers are needed to identify febrile seizure patients at risk of SUD. (Holm IA et al. Pediatr Neurol 2012 April;46:235-239).

\title{
READING EPILEPSY RESPONSE TO ANTICONVULSANTS
}

Investigators at the Department of Neurology, Vanderbilt University Medical Center, Nashville, TN report 3 patients with reading epilepsy, a boy aged 14 years, a 26year-old male, and a 27-year-old woman, 2 having an excellent response to levetiracetam. The boy had tremors in the jaw and tongue when reading silently, and these progressed into loss of consciousness and generalized jerking if reading was continued. These episodes did not occur when reading aloud, skimming material, or doing mathematics. Video-EEG showed bifrontal synchronous sharp waves or spike-and-wave complexes, occasionally becoming generalized with left predominance. MRI was normal. He was treated effectively with divalproex sodium. Tremors did not recur when divalproex was inadvertently discontinued at 3 years after seizure onset, and at 6-year follow-up he was still seizure-free and on no medication. The 2 adults had twitching of the right lips or "mouth jumping" while reading. One had a video-EEG-monitored, generalized tonicclonic seizure with left fronto-cental predominance, resistant to lamotrigine and controlled with levetiracetam; the other had a complex partial seizure with left mesial temporal sclerosis. Following left amygdalohippocampectomy, the reading epilepsy was partially controlled with carbamazepine and fully controlled with levetiracetam. Levetiracetam is proposed as first-line treatment for primary and secondary reading epilepsy. Spontaneous medication-free remission of primary reading epilepsy may occur within 3 years of seizure onset. (Haykal MA, El-Feki A, Sonmezturk HH, Abou-Khalil BW. New observations in primary and secondary reading epilepsy: excellent response to levetiracetam and early spontaneous remission. Epilepsy Behav 2012 April;23:466-470). (Respond: Dr BW Abou-Khalil. E-mail: bassel.abou-khalil@vanderbilt.edu). 
COMMENT. Reading epilepsy, a form of reflex epilepsy, first described by Bickford R at the Mayo Clinic in 1956, occurs in 2 forms, primary or idiopathic and secondary and attributed to a structural brain lesion. Levetiracetam may prove superior to valproate and clonazepam in treatment, and spontaneous remission of primary reading epilepsy may occur in idiopathic cases. Generalized tonic-clonic seizures may be avoided by stopping reading at onset of mouth jerking, but anticonvulsant medications are usually required. The left predominance of the EEG seizure discharge in cases of reading epilepsy is in keeping with reports of reduced white matter integrity in the left arcuate fasciculus of dyslexics. (Vandermosten M et al. Brain 2012;135:935-948; see Ped Neur Briefs 2012 April;26(4):32).

Neurocognitive endophenotype with rolandic epilepsy. Children with rolandic epilepsy (RE) have reading, language, and attention disorders. In 13 probands with RE and 11 epilepsy-free siblings who completed a neurocognitive evaluation, $9 \%$ of siblings and $31 \%$ of probands were reading impaired, $36 \%$ of siblings and $54 \%$ of probands were language impaired, and $70 \%$ of siblings and $67 \%$ of probands had attention impairments. Probands and siblings showed a similar profile of deficits in language and attention. Early psychological evaluation and academic intervention may benefit children with RE. (Smith AB, et al. Epilepsia 2012 March;53(4):705-711).

\section{INFLAMMATORY DISORDERS}

\section{HEMOPHAGOCYTIC LYMPHOHISTIOCYTOSIS}

Investigators at the Hopital Bicetre and other hospitals in Paris, France studied the CNS symptoms at onset of primary hemophagocytic lymphohistiocytosis (HLH), and differentiated these from other CNS inflammatory diseases. At disease onset, 46 patients included in the study had a median age of 2.5 months (range 0-190 months), and 34 (74\%) were under 12 months. Familial HLH was the most frequent genetic defect. Neurologic symptoms were present at onset in 29 children (63\%) and were associated with fever, hepato-splenomegaly or lymphadenopathy in 26 . The 3 main neurologic symptoms were seizures, impaired consciousness, and meningismus. Microcephaly was diagnosed at birth in 6 boys and developed shortly after birth in 1. CSF was abnormal in $50 \%$. MRI was abnormal in $7(15 \%)$. Unlike patients with ADEM, MRI showed symmetric periventricular lesions, without thalamic and brainstem involvement and with infrequent hyposignal intensity on T1. At end of follow-up (3.6 +/- 3.6 years), 18 (39\%) patients had died; 17 of 28 (61\%) surviving patients were normal neurologically, $5(18 \%)$ had a severe neurologic outcome, and $6(21 \%)$ had mild cognitive deficits. Risk of abnormal neurologic outcome was related to neurologic symptoms, MRI abnormalities, or abnormal CSF at onset, and was not influenced by age or type of genetic defect. (Deiva K, Mahlaoui N, Beaudonnet F, et al. CNS involvement at the onset of primary hemophagocytic lymphohistiocytosis. Neurology 2012 April 10;78:1150-1156).

(Response and reprints: kumaran.deiva@bet.aphp.fr).

COMMENT. Neurologic symptoms are frequent at onset of primary HLH and $50 \%$ patients have abnormal CSF. Early diagnosis is essential for a favorable outcome. 